\title{
Bright enough to see atoms?
}

\author{
A.L. Bleloch ${ }^{*}$, U. Falke ${ }^{*}$ and U. Bangert ${ }^{* *}$ \\ *SuperSTEM laboratory, CLRC Daresbury, WA4 4AD, UK \\ ** Department of Materials Science, Manchester University, Manchester, UK
}

Now that sub-angstrom resolution is becoming routinely accessible, we need to critically assess which materials problems can now be tackled that could not be tackled hitherto. Understandably, we progress by first showing that the new generation of instruments indeed confirms structures we already know (only more emphatically) but to justify the considerable expense, we need to be clear what new science will become accessible. Here we will discuss the detection of single dopant atoms in a solid by EELS alone. This is a benchmark experiment that defines new and useful experiments accessible only with high brightness stable aberration corrected instruments.

A large and growing range of functional materials rely for their utility on the influence of isolated atoms or point defects consisting of a complex of isolated atoms with vacancies. The most obvious example is doping of semiconductors but the phenomenon extends to sub atomic layer segregation, to grain boundaries in structural materials and to platinum group metal atoms in heterogeneous catalysts. Previously isolated dopant atoms have been detected by Muller et al. by high angle annular dark field (HAADF) imaging and by Varela et al. by a combination of imaging and EELS $[1,2]$. HAADF (and phase contrast imaging) rely on scattering phenomena that do not intrinsically provide an unambiguous signature for an atomic species i.e. a number of atoms of one species could produce the same signal as one atom of another species. This means that either a priori assumptions about the sample are required or a spectroscopic signal is needed. Spectroscopy allows one species of atom to be selected out from a background of other atoms

If we wish to detect by the EELS signal alone a single atom of one atomic species in a practical sample, we need to ensure that there will be sufficient signal available and that the signal is not swamped by the background. Consider one atom of phosphorous in a column of 100 silicon atoms. For the sake of this argument, an approximate cross section for the EELS signal is $10^{-25} \mathrm{~m}^{2} / \mathrm{eV}$. In this case, for a VG HB501 with a Nion corrector giving a 100pA $100 \mathrm{keV}$ electron beam with a $24 \mathrm{mrad}$ convergence angle, in a collection time of $1 \mathrm{~s}$ we can expect a signal to noise of 10 for a probe of $0.1 \mathrm{~nm}$. In a previous generation instrument, we could expect a signal to noise of 0.1 for a probe of $1 \mathrm{~nm}$. That is two orders of magnitude difference in signal-to-noise for a one-order change in probe size. This is because the target signal (i.e. the P) goes up as the current is concentrated in a smaller probe and simultaneously, the background goes down because there is less current exciting the background atoms. This order-of-magnitude calculation demonstrates that probe size has a very profound influence on single atom detection. What is less obvious is that source brightness is, in fact, the limiting experimental constraint. If the collection time goes up by a factor of 10 because the probe current in the $0.1 \mathrm{~nm}$ probe is $0.01 \mathrm{nA}$ rather than $0.1 \mathrm{nA}$, then the stability requirements become infeasible. That is, the practical source size (or brightness) should include instabilities and drift. Fig. 1 shows a bright field phase contrast image of a carbon nanotube which has been ion implanted with Mn. Fig 2 shows spectra from adjacent points with a signal consistent with one atom of $\mathrm{Mn}$. These data are marginal because not all instabilities have been rooted out and therefore the effective 
probe size is broadened over the integration time. Also, the channel-to-channel gain variation has not been optimally normalised out.

Single atom detection is the hallmark experiment of the aberration correction generation of STEM instruments and promises a fertile field of scientific problems to address.

\section{References}

[1] P. M. Voyles et al., Nature, 416 826-829 (2002)

[2] M. Varela et al., Phys. Rev. Lett. 92, 095502 (2004)
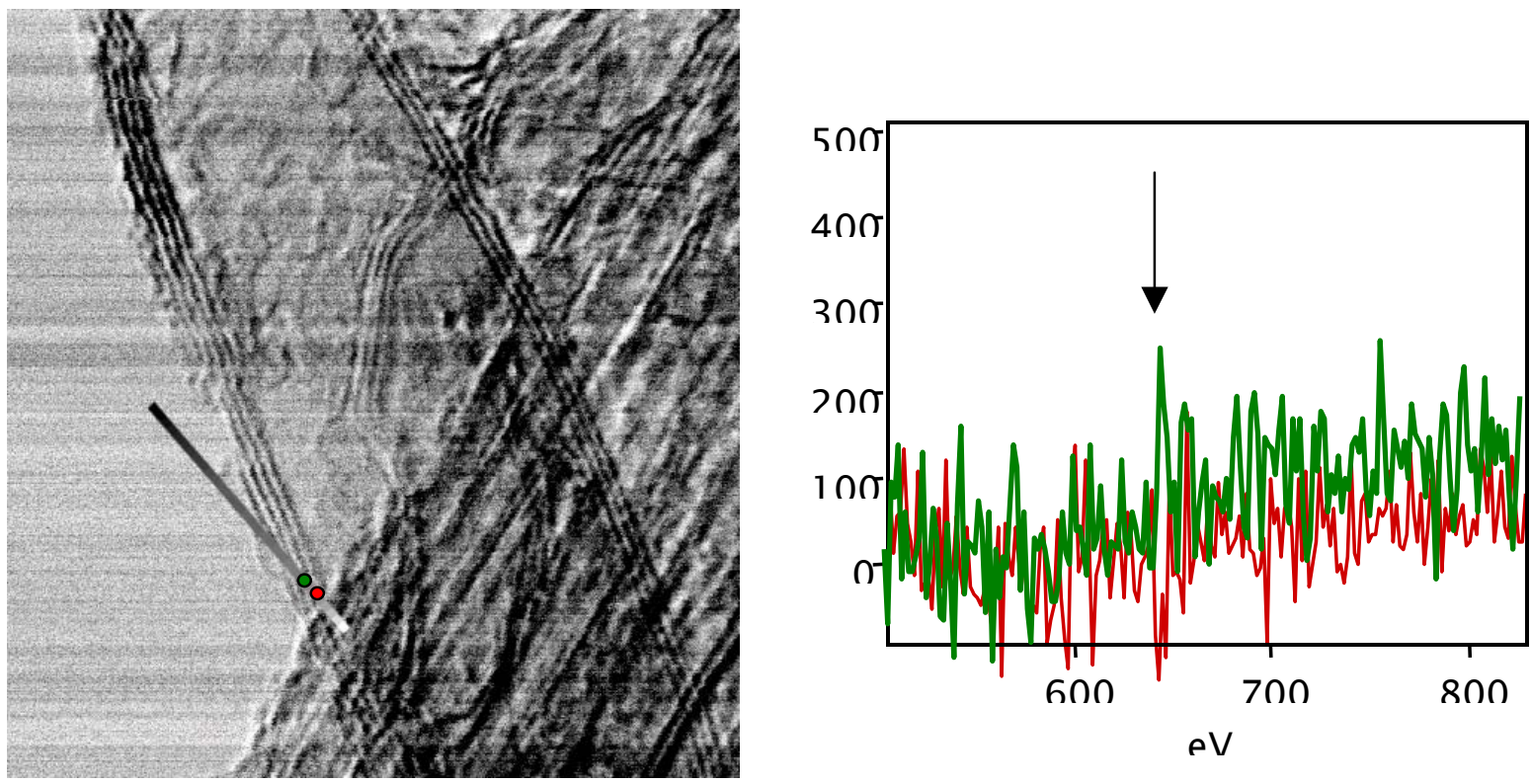

Fig. 1 A multiwall Mn doped carbon nanotube. Electron energy loss spectra were obtained along the diagonal line shown.
Fig. 2. Two adjacent spectra from the nanotube wall. The arrow shows the onset of the Mn edge. The bold spectrum comes from the left dot indicated in Fig 1. 